Tra romanzo e autobiografia: narratore e autore (si) confondono

Elisabetta Santoro ${ }^{1}$

\title{
Abstract (En/lt)
}

The last novel of Julio Monteiro Martins, a Brazilian writer who lives and writes in Italy and in Italian, is entitled Madrelingua (Besa Editore, 2005). Besides the characters who give life to the novel, a first and a second narrator emerge in the book. The last one always appears between brackets and makes comments in the first person which, under various aspects, recall biographical data about the writer. The reader gets 'confused': in the end, s/he does not know who is speaking and can overlay the figure of this second narrator on to that of the author. In this article we propose a reflection on the meaning that such a choice can have from an 'autobiographical' point of view. The 'I' reveals and hides itself and a tenuous line marks off and, at the same time, allows a crossing, offering the possibility going beyond the boundaries of a single literary genre.

Si intitola madrelingua (Besa Editore, 2005) l'ultimo romanzo pubblicato da Julio Monteiro Martins, uno scrittore brasiliano che vive e scrive in Italia e in italiano. Oltre ai personaggi che danno vita al romanzo, entrano in scena nel libro un primo e un secondo narratore. Quest'ultimo compare sempre tra parentesi quadre e fa commenti in prima persona che per molti aspetti ricordano dati biografici dello scrittore, 'confondendo' in tal modo il lettore che finisce per non sapere chi sta parlando e per sovrapporre la figura di questo secondo narratore e quella dell'autore. In questo testo ci proponiamo di riflettere sul significato che una tale scelta può avere da un punto di vista 'autobiografico', visto che l'io si rivela e si nasconde e che una tenue linea separa e, al tempo stesso, permette un attraversamento che offre la possibilità di andare oltre $\mathrm{i}$ confini di un solo genere letterario.

L'oggetto del presente lavoro è l'ultimo romanzo di Julio Monteiro Martins, intitolato madrelingua e pubblicato nel $2005^{2}$. Immaginiamo, per iniziare, un ipotetico lettore che prenda in mano il libro per la prima volta: osservando la copertina (che abbiamo qui riprodotto), vediamo che gli si presentano, già a partire da questa parte del testo, diversi spunti e aspetti da esaminare.

\footnotetext{
${ }^{1}$ Universidade de São Paulo.

${ }^{2}$ Julio M. MARTINS, madrelingua, Lecce, Besa Editore, 2005.
} 


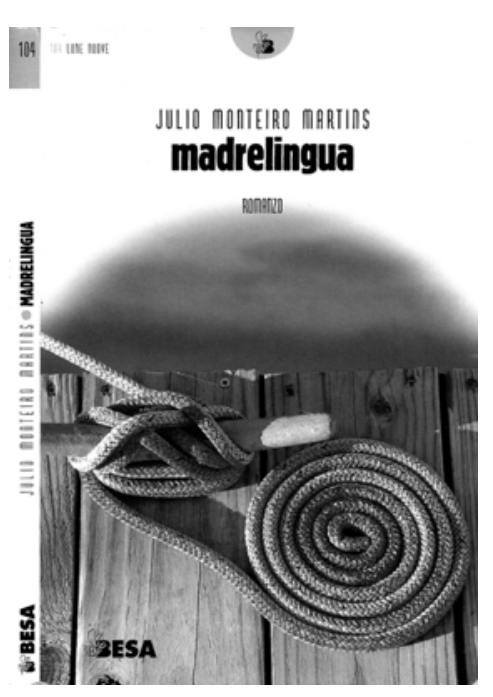

Limitandoci ai soli elementi verbali, possiamo intanto prevedere che questo lettore si potrebbe soffermare sul nome dello scrittore (il primo elemento che compare in alto). Julio Monteiro Martins è un nome non 'classicamente' italiano e fa, quindi, immediatamente pensare a uno scrittore straniero. Se la curiosità del nostro lettore lo portasse a voler sapere se il libro è tradotto o se Martins è parte dell'ormai piuttosto numerosa schiera di scrittori non italiani che vivono in Italia e scrivono in italiano, basterebbe uno sguardo al risvolto di copertina per scoprire che Martins è brasiliano, di Niterói, nello stato di Rio de Janeiro, ma che, pur continuando a trascorrere lunghi periodi in Brasile, vive da ormai più di quindici anni in Italia, dove, oltre ad insegnare lingua portoghese e traduzione letteraria all’Università di Pisa, si dedica anche al laboratorio di narrativa della Scuola Sagarana, dirige la rivista con lo stesso nome e, soprattutto, scrive racconti e romanzi, attività che non è iniziata nel periodo in cui si è trasferito in Italia, ma che già svolgeva con una certa regolarità anche in Brasile.

Queste notizie cominciano a stimolare anche alcune ipotesi sul possibile significato del titolo del libro che si legge sulla copertina subito dopo il nome: madrelingua. Per chi sa che l'autore di questo libro sta scrivendo in una lingua straniera, anche quest'aspetto può suscitare osservazioni interessanti, poiché quello della lingua è un tema fondamentale per chi scrive da straniero in un paese straniero e adotta, quindi, la lingua del posto che lo ospita per poter essere letto dai suoi 'connazionali d'adozione'. Nel caso di Martins le due lingue in questione sono il portoghese brasiliano, la sua madrelingua appunto, e l'italiano, la lingua del paese in cui vive e lavora e al 
quale costantemente si riferisce anche nel suo libro, pur senza mai dimenticare il Brasile. Questa commistione fra l'Italia e il Brasile è sempre presente anche nella lingua, nelle scelte tematiche e nel modo di esprimersi e accompagnerà chi legge lungo tutta l'opera, dando origine a un singolare risultato che ricorda costantemente la duplice appartenenza.

Le considerazioni fatte fin qui bastano ad accorgersi che sono molteplici gli spazi per la riflessione che il libro offre. In questo testo ci concentremo, tuttavia, in particolar modo, sul tema introdotto dal terzo elemento verbale della copertina. Di fatto, dopo il nome dell'autore e il titolo dell'opera si legge romanzo, e anche questa è una 'affermazione' essenziale per poter completare le aspettative di chi si appresta ad aprire un libro e a leggerlo. Rifletteremo, dunque, su come il libro è stato strutturato e vedremo che, da un certo punto di vista, nonostante si affermi più volte che si tratta di un romanzo, si potrebbe parlare forse anche di autobiografia. $\mathrm{Ci}$ domanderemo se e come si possano conciliare questi due generi, partendo da una problematizzazione del ruolo del narratore e di quello dell' 'autore', cercando di individuare quali sono i limiti tra l'uno e l'altro e facendo ipotesi che ci portino a pensare a come analizzare e cercare di capire le conseguenze di un'operazione di questo tipo.

Romanzo, dicevamo e potremmo continuare chiedendoci: che cosa ci si aspetta quando uno scrittore fa un'evidente 'dichiarazione d'intenzioni' dicendo sin dalla copertina che quello che ha scritto e pubblicato è un romanzo? Se è vero che sono molteplici le sue definizioni ${ }^{3}$, è anche vero che tutte hanno almeno un aspetto in comune: un romanzo è un racconto con argomento fantastico (ovvero, di invenzione) che parte dall'immaginazione di chi lo scrive e che non ha nessun tipo di impegno nei confronti della realtà e della verità. Il romanzo viene costruito a partire da personaggi e intrecci che confluiscono in una storia presentata da un narratore, che la può raccontare in prima o terza persona, che può essere o no parte della storia stessa (può essere, cioè, un narratore intra o extradiegetico), che può scegliere un unico punto di vista o adottarne diversi, che sa ogni dettaglio della storia, compreso ciò che pensano i personaggi (è, cioè, un narratore onnisciente) o che invece la racconta sulla base delle sole informazioni di cui nel suo ruolo 'verosimilmente' potrebbe essere in possesso.

Che queste siano alcune tra le principali caratteristiche di un romanzo, Julio Monteiro Martins di certo lo sa. Di fatto, nella prima parte del libro, che lo scrittore chiama 'Preambolo' e che precede l'inizio della narrazione vera e propria, mostra subito la sua familiarità con i temi che

\footnotetext{
${ }^{3}$ Un riferimento interessante può essere, tra le tante altre, l'opera intitolata L'universo del romanzo di Bourneuf e Ouellet che, oltre ad un'approfondita introduzione, contiene capitoli riguardanti: la storia e la narrazione; il punto di vista; lo spazio; il tempo; i personaggi; il romanzo e il suo autore (Roland BouRNEUF \& Réal OUELLET, L'universo del romanzo, Torino, Einaudi, 1976).
} 
riguardano questioni teoriche della scrittura e, in una descrizione metaletteraria proprio sul genere prescelto, dice che ai nostri giorni «non è più possibile scrivere un romanzo, e non è più possibile non scriverlo» (p. 14), rivolgendo un'attenzione particolare ai romanzi 'lasciati a metà', come il suo madrelingua, che si dovrebbe inserire nella tradizione dei romanzi incompiuti, ai quali la storia letteraria ha dedicato, secondo lui, poca o nessuna attenzione. Oltre a quest'osservazione, nel «Preambolo» Martins presenta il suo libro e lo annuncia dicendo che è «[...] la storia di un altro romanzo incompiuto, che dovrebbe chiamarsi madrelingua (proprio così, con la ' $\mathrm{m}$ ' minuscola). O meglio, è quello stesso romanzo, il suo percorso tortuoso, fino al momento della sua irrimediabile sospensione, inframmezzato di commenti e di confessioni tra parentesi quadre» ${ }^{4}$.

Martins ci presenta, insomma, un tipo particolare di romanzo, consapevole però di qual è il suo punto di partenza e di quali sono i suoi riferimenti. L'autore ci prepara alla lettura di una storia che si interrompe, che finisce prima del tempo, mentre ci viene annunciata, sin da queste pagine del «Preambolo», la presenza 'di commenti e di confessioni tra parentesi quadre'. Su quest'aspetto torneremo fra poco. Nel frattempo, vale forse la pena di ricordare che subito dopo quest'affermazione, Martins presenta il protagonista del romanzo che sta per iniziare, Mané, pregando i suoi lettori di non affezionarsi troppo né a lui, né agli altri personaggi e continua con la descrizione di un non meglio identificato scrittore, di cui annuncia la crisi che verrà narrata nel romanzo, aggiungendo fra parentesi un commento sulla natura dello 'scrittore migrante' e sull'adeguatezza del romanzo incompiuto per uno scrittore di questo tipo.

È inevitabile associare quanto viene affermato a Martins stesso - ovvero all'autore ontologico che è dietro la 'penna' - e credere che stia parlando della sua condizione personale di scrittore brasiliano in Italia, cosa che fino a questo punto è eventualmente ancora lecito che faccia, visto che il romanzo non è ancora iniziato e che, quindi, il patto narrativo e la finzione possono essere ancora considerati 'in preparazione'. Interessante è osservare, comunque, che Martins rinuncia ad uno dei più utilizzati stratagemmi per rafforzare la presenza dell'autore nel testo, ovvero, alla firma del testo stesso. Leggere alla fine del «Preambolo» lo stesso nome che si è letto in copertina avrebbe sicuramente avuto la conseguenza di 'far credere' in maniera ancora più univoca all'effettiva identità e coincidenza tra chi scrive e colui di cui si parla. Ma vediamo cosa succede dopo.

Arrivati alla pagina successiva, leggiamo ancora una volta il titolo del romanzo che domina da solo sul foglio altrimenti bianco e siamo portati ad interpretarlo come il segnale del vero e

\footnotetext{
${ }^{4}$ Julio M. MARTINS, madrelingua, op. cit., p. 13.
} 
proprio inizio della storia, convinti di poterci preparare a entrare nel mondo della finzione e dell'immaginazione che lo caratterizzano. Giriamo la pagina e siamo subito sorpresi dalle 'parentesi quadre' nei confronti delle quali eravamo già stati messi in guardia: sono le parentesi quadre che, stando a quanto ci ha detto lo stesso scrittore, inframmezzeranno il libro 'di commenti e di confessioni'.

La prima frase che leggiamo sembra effettivamente un commento: [Con questo dialogo piuttosto familiare inizia madrelingua: $]^{5}$ che viene da un'istanza che non è né dentro al romanzo, né fuori da esso e che comunque ci impedisce di lasciarci subito prendere dalla storia raccontata. Seguono due battute al discorso diretto e, poco più avanti, un pronome personale ('mi rispose'), un aggettivo possessivo ('La mia piccola storia') e verbi di prima persona singolare ('ero nato'; 'sarò diventato') rivelano che la storia è narrata da un io che subito dopo si presenta dicendo il suo nome 'Manoel Alves dos Santos, detto, Mané', la sua età ('sessant'anni'), il suo luogo di nascita ('Niterói'), la città italiana in cui è andato a vivere ('Firenze') e il periodo in cui l'ha fatto, identificato attraverso il cognome del Presidente del Consiglio dell'epoca ('periodo Craxi'). È nel momento di questa presentazione che scopriamo che la voce che parla tra parentesi quadre è praticamente la voce di un secondo narratore che non dice il suo nome, ma dà - come in una sorta di 'controcanto' - tutte le altre informazioni su di sé subito dopo quelle di Mané, portando ad un inevitabile confronto con lui. È così che il lettore sa che se Mané ha sessant'anni, questo 'secondo narratore' ne ha 46, che è anche lui di Niterói, che non è andato a vivere a Firenze, ma a Lucca e che è arrivato in Italia nel 'periodo Dini' invece che nel 'periodo Craxi'.

Abbiamo, cioè, un secondo io narrante che accompagna il primo, commenta le sue scelte e finisce in alcuni momenti per sovrapporsi alle sue parole appropriandosi, con un atteggiamento spesso irriverente, dello spazio del romanzo. Il suo comportamento cambia nel corso del libro e i suoi interlocutori si alternano, in modo che, se a volte sembra rivolgersi direttamente al primo narratore Mané e a volte sembra invece parlare con il lettore, altre volte si rivolge a se stesso e, altre volte ancora, perfino a lettori specifici o terzi. Vediamo degli esempi.

A pagina 29 leggiamo: "Come avrei potuto resistere, per di più annebbiato dalla batida de coco degli argentini? [ti capisco]» e la presenza del pronome di seconda persona singolare che segue una domanda in prima persona del narratore Mané ci permette di identificare che il dialogo che si stabilisce è tra loro e che il lettore ne resta apparentemente fuori, anche se l'atteggiamento ironico del secondo narratore, evidenziato da affermazioni di questo genere, dà

${ }^{5}$ Ibidem, p. 17. 
indizi a chi legge sul 'tono di voce' di questo narratore e contribuisce alla costruzione di una sua immagine.

Il 'dialogo' non resta però sempre tra i due narratori. In altri casi è evidente che la spiegazione, il commento o la didascalia del secondo narratore si rivolgono a lui stesso e più o meno direttamente al lettore, cercando di allontanarlo dalla finzione del romanzo e di spingerlo a credere che la voce che entra in scena tra parentesi quadre coincide con l'autore in carne ed ossa, come quando scrive:

Prima di K43 la mia ragazza era una belva rossa torinese, figlia di inglesi, di nome Miranda [1'ho presa tutta dalla mia ex fidanzata Sheila, quando avevo 19 anni e lei 20, forse la donna più bella e selvaggia che abbia mai avuto $]^{6}$.

oppure

Oltre il confine dei sogni, come un disperato delirio, c'è un mondo tempestoso, ci sono tuoni, fulmini. Chiudo le finestre, ma come un ariete invisibile, elettromagnetico [cosa?], quella tempesta vuole entrarmi in casa attraverso la radio, la tv, la rete [ah, ora ho capito la metafora] [una parentesi nella parentesi: so che non sono molto simpatici questi commenti in mezzo al testo, ma non resisto proprio: quando viene, viene. Va bé, cercherò di controllarmi un po'] $[\ldots]^{7}$

È quasi inevitabile che affermazioni di questo genere portino il lettore a uscire momentaneamente dal suo ruolo di fruitore di una storia inventata per trasformarsi in 'confidente' al quale vengono rivelati particolari presentati come se riguardassero tanto la costruzione del romanzo, quanto la vita di chi lo scrive, che appare come fonte d'ispirazione di personaggi e situazioni. Il secondo narratore parla a se stesso e al lettore in un gioco in cui a prima vista 'scopre' se stesso e mostra i suoi segreti attraverso tutta una serie di dettagli sul processo di stesura del libro, che lo legano in modo sempre più evidente ad una vita che sembra vissuta e che sembra 'realtà'.

E c'è ancora un altro modo di usare lo spazio racchiuso tra le parentesi quadre: in alcuni casi il secondo narratore cita una persona in particolare che non fa parte del gioco narrativo (non è il narratore, non è un personaggio e non è il lettore) e che, quindi, da una parte, esclude il 'lettore

\footnotetext{
${ }^{6}$ Ibidem, p. 33.

${ }^{7}$ Ibidem, p. 19.
} 
comune' poiché si rivolge, invece, ad un lettore specifico che non siamo tenuti a riconoscere, e dall'altra, svela un dettaglio personale come il nome di un'amica. È quello che succede in quest'esempio: «[...] ha lasciato nell'aria un profumo di magnolia che mi ha fatto girare per seguirla lungo Por Santa Maria fino all'ingresso degli Uffizi [grazie, Mia, per quella mappa di Firenze e per le indicazioni] ${ }^{8}$.

È così che si sviluppa nel libro la presenza del 'commentatore' (p. 14) e il meccanismo, annunciato, come dicevamo, sin dalle prime pagine, continua fino all'improvvisa interruzione della storia, anch'essa resa 'ufficiale' da un lungo testo fra parentesi quadre, seguito da un altro testo che è una 'parentesi nella parentesi' in cui si narra la morte del padre del secondo narratore con strategie come l'uso di crononimi, toponimi e antroponimi che contribuiscono alla costruzione di una scrittura che fa di tutto per sembrare 'reale' e indurre il lettore ad identificarla con la 'verità. Di fatto, si legge, per esempio, una data precisa che áncora fortemente l'episodio narrato alla realtà (7 settembre 2002) e si continua con indicazioni di luoghi concreti (il Brasile, la spiaggia di Icaraí, la casa, il lettone, ...), di nomi propri (Lorenzo), di ricordi riportati con dovizia di particolari. Ecco, dunque, che il romanzo sta per finire e leggiamo:

[ma prima un (sic) parentesi nella parentesi: proprio mentre scrivevo queste righe - era il pomeriggio del 7 settembre 2002 - è morto mio padre. Ho ricevuto una telefonata dal Brasile la mattina dopo. Lorenzo mi dormiva accanto, sul lettone, e non si è svegliato. Avevo la sua stessa età, sei anni, quando sulle sabbie bianchissime della spiaggia di Icaraí guardavo mio padre che saltava dalla piattaforma più alta di un grande trampolino di cemento a forma di ' $v$ ', in mezzo al mare. Ero sempre circondato da belle ragazze affascinate, che mi chiedevano quale fosse il nome del mio papà, e lo applaudivano quando saltava. Ero fiero di lui, di avere un padre così bello e coraggioso. $[\ldots]^{9}$.

È questa l'ultima inserzione del secondo narratore che, come abbiamo visto, compare da subito, interloquisce in diversi modi e, secondo il «Preambolo», è l'autore della versione precedente del romanzo, ovvero il narratore stesso, cosa che verrebbe confermata quando a pagina 33 tra parentesi quadre leggiamo: «[Mané. Ossia, io]».

A romanzo praticamente finito, le parentesi quadre del secondo narratore tornano ancora in un post scriptum (introdotto dall'abbreviazione PS), in cui si narra un ultimo episodio 'reale' e cioè

\footnotetext{
${ }^{8}$ Ibidem, p. 34.

${ }^{9}$ Ibidem, p. 63.
} 
la reazione di due amici di fiducia dell' 'autore' che, dopo aver letto la prima bozza del libro, avrebbero espresso alcune preoccupazioni tra cui proprio quella riguardante l'identità del narratore che non risulta chiara e «viene a confondersi con quella racchiusa nelle parentesi quadre $[\ldots] \gg{ }^{10}$.

Di fatto, il secondo narratore, come il primo, dice io e racconta fatti che riguardano la sua vita 'personale' tanto in Italia, quanto in Brasile che sembrano coincidere - o almeno potrebbero coincidere - con la vita 'reale'. Tra le altre cose, questi episodi vengono raccontati retrospettivamente e collegati al momento presente attraverso scelte di tempi verbali che privilegiano il passato prossimo e l'imperfetto, in modo da creare nei confronti di chi legge l'illusione di un racconto in presenza in cui le storie, riviste alla luce del presente, assumono contorni reali e assomigliano sempre di più a confidenze intime.

Si confonde così il lettore che non resiste alla tentazione di credere che Julio Monteiro Martins, ossia il 'vero' autore citato in copertina, stia scrivendo di se stesso, fino ad arrivare perfino al racconto della morte di suo padre. Sembrerebbe chiaro che si assiste ad un procedimento narrativo che dal 'romanzo annunciato' si sposta progressivamente verso un' 'autobiografia', soprattutto se vogliamo considerare tale, come fa Misch, qualsiasi opera in cui si possano scoprire fatti della vita di colui che narra: «Può essere definita solo riassumendo ciò che implica il termine 'autobiografia' - la descrizione (graphia) di una vita umana individuale (bios) fatta dall'individuo stesso (auto)» ${ }^{11}$.

Ciò non basterebbe, tuttavia, a parlare di autobiografia se, come invece afferma Lejeune, si volesse considerare che, oltre a questo, è necessario che l'autobiografia, per potersi dire tale, contenga il cosiddetto 'patto autobiografico', che è il presupposto tramite il quale Lejeune cerca di risolvere, tra le altre cose, la questione della distinzione tra romanzo e autobiografia, altrimenti praticamente impossibile da chiarire in maniera definitiva. Perché si possa parlare di autobiografia, bisognerà allora avere segnali più univoci come la ripetizione dello stesso nome per indicare autore, narratore e personaggio, un titolo che affermi chiaramente che si sta leggendo un'autobiografia e, soprattutto, un chiaro accordo tra 'autore' e 'lettore', a partire dal

\footnotetext{
${ }^{10}$ Ibidem, p. 64.

${ }^{11}$ «It can be defined only by summarizing what the term 'autobiography' implies - the description (graphia) of an individual human life (bios) by the individual himself (auto)» (Georg MiscH, A History of Autobiography in Antiquity, Londra, Routledge, p. 5).
} 
quale si costruisce il loro rapporto letterario che deve essere di fiducia reciproca e basarsi su un criterio referenziale ${ }^{12}$ perché tratta di una realtà esterna al testo e, quindi, passibile di verifica.

Ebbene, tutto questo in madrelingua non c'è: non c'è un titolo che faccia riferimento all'autobiografia, il genere indicato è il romanzo e non c'è un nome che identifichi univocamente il secondo narratore. Al contrario, nel libro di Martins il secondo narratore è semplicemente un io. Se, però, è vero, come afferma Benveniste, che «è io chi dice io» ${ }^{13}$ il procedimento narrativo escogitato non solo permette di giocare con il detto e il non-detto e con il rapporto tra realtà e finzione, ma amplia il raggio d'azione della scrittura poiché consente di costruire mondi diversi senza i limiti normalmente imposti da precise categorizzazioni. La presenza di un secondo narratore tra parentesi quadre, discussa e programmata sin dal «Preambolo», sarebbe, dunque, uno stratagemma con un obiettivo specifico, grazie al quale si possono mescolare vite reali e immaginate, si possono alternare effetti di senso di avvicinamento e allontanamento, si può passare dalla soggettività all'oggettività e si possono, in ultima istanza, rompere i confini tra due generi letterari. Il lettore viene portato a penetrare sempre più in una manovra di scrittura, in cui non si sa cosa leggere come parte del romanzo e cosa, invece, considerare racconto della vita 'reale' e, nonostante il tema ufficiale di madrelingua non sia il racconto autobiografico dell'autore, si ha l'impressione che gli episodi della sua vita prendano via via il sopravvento e spingano a maturare la convinzione che, in realtà, l'autore stia parlando di se stesso e stia, quindi, in qualche modo, scrivendo una sorta di autobiografia nella quale, però, si rivela e si nasconde.

Di fatto, un'altra essenziale differenza di madrelingua rispetto a un'autobiografia 'classica' è che, mentre di solito l'io parla di se stesso ed è contemporaneamente tanto il soggetto del presente quanto il soggetto del passato, in questo testo lo stratagemma creato sdoppiando l'io e affidando la narrazione a due figure separate e, al tempo stesso, unite permette che l'io del presente dialoghi con l'io del passato, facendo penetrare nel romanzo il racconto della sua 'vita umana individuale'.

Se cerchiamo ora di pensare alle principali differenze di composizione tra il romanzo e l'autobiografia (escludendo quindi la questione che il romanzo è 'finzione', mentre l'autobiografia è 'reale'), la conclusione a cui giungiamo è che, mentre nel romanzo il narratore e il personaggio non devono necessariamente coincidere e, soprattutto, entrambi di certo non si

\footnotetext{
${ }^{12}$ Cf. Philippe LeJeunE, Le pacte autobiographique, Parigi, Seuil, 1975, p. 36.

${ }^{13}$ Il riferimento è al saggio di Émile Benveniste intitolato «La soggettività nel linguaggio» e pubblicato in Problemi di linguistica generale, (Milano, Il Saggiatore, 1994, pp. 310-320), la cui prima traduzione in italiano risale al 1971.
} 
confondono con il cosiddetto 'enunciatore' (inteso - seguendo la linea teorica della semiotica del testo e del discorso ${ }^{14}$ - come istanza presupposta all'enunciato, che non corrisponde all'autore ontologico); nell'autobiografia, invece, succede che il narratore deve necessariamente identificarsi con il personaggio (ovvero, deve esserci un io che parla di se stesso), creandosi in tal modo l'illusione che entrambi coincidano con l'enunciatore e con l'autore 'vero', vale a dire, colui che firma il testo e che dichiara di averlo scritto. Avremo, quindi, una situazione di questo tipo:

\begin{tabular}{|c|c|}
\hline ROMANZO & AUTOBIOGRAFIA \\
\hline Narratore $\neq$ personaggio $\neq$ enunciatore $\neq$ autore & Narratore $=$ personaggio $=$ enunciatore $=$ autore \\
\hline
\end{tabular}

Pensare in questi termini al romanzo e all'autobiografia ci aiuta a riflettere sull'operazione narrativa compiuta nel libro che stiamo analizzando. In madrelingua è come se entrambi i generi fossero presenti e si appropriassero di una parte del libro, visto che abbiamo, da una parte, un narratore in prima persona, Mané, che è anche il personaggio principale del romanzo e che narra la sua storia, presentata come storia fittizia e, dall'altra, il secondo narratore che si muove su un piano estraneo alla storia narrata e che usa lo spazio tra parentesi quadre per raccontare altre storie (le 'sue' storie) e 'far credere' che ciò che racconta è vero, appartiene alla sua vita vissuta ed è in parte stato fonte d'ispirazione per il romanzo.

In altre parole, Mané sarebbe il narratore-personaggio che non coincide con l'enunciatore, mentre il secondo io si appropria di uno spazio creato al fine di far subentrare altri racconti, che altrimenti sarebbero rimasti al di fuori del romanzo, e così facendo inserisce nel libro nuove prospettive di lettura e giochi temporali che contribuiscono a ridurre le barriere tra un genere e l'altro e tra un io e l'altro, fino ad abbatterle del tutto, quando si mette in scena la loro identità (come nell'affermazione già citata [Mané. Ossia, io ${ }^{15}$ ).

\footnotetext{
${ }^{14}$ Per un'introduzione in italiano alla teoria semiotica fondata da Algirdas Julien Greimas a cui ci riferiamo qui rimandiamo all'opera Semiotica del testo di Maria Pia Pozzato (Roma, Carocci, 2001) e al Dizionario scritto da Greimas stesso e da Jóseph Courtés (Algirdas Julien GreIMAS \& Joseph COURTÉS, Semiotica: dizionario ragionato della teoria del linguaggio, Firenze, La Casa Usher, 1986).

15 J. M. MARTINS, ibidem, p. 33.
} 
E. Santoro: Tra romanzo e autobiografia: narratore e autore (si) confondono

In madrelingua la soluzione per poter rientrare in entrambi i generi - e, allo stesso tempo, in nessuno dei due - è, quindi, sovrapporre storie e piani narrativi, permettendo a Mané di raccontare la sua storia e di gestire i suoi personaggi, mentre la vita di chi scrive viene 'apparentemente' rivelata attraverso il secondo narratore che controlla uno spazio sempre maggiore e si prende la libertà di cambiare e di trasformare il suo rapporto con le altre istanze che fanno parte del romanzo, creando l'illusione della realtà e confondendo i due narratori, il rapporto tra narratore e autore, il lettore, le storie e i generi.

È a questo punto importante riflettere sul ruolo dell'enunciatore, al quale abbiamo già accennato poc'anzi. Guardare i testi letterari a partire dall'idea che colui che ci immaginiamo come autore del testo non corrisponde mai all'autore ontologico significa poter esercitare un maggiore distanziamento nei confronti dei meccanismi di costruzione del testo e del discorso che verranno esaminati per il valore e per la funzione che rivestono all'interno dello stesso testo. È così che la semiotica, invece di interrogarsi sull'autore 'reale', cerca indizi che permettano di ricostruire l'ethos di un enunciatore, il suo tono di voce e il suo modo di stare nel mondo. Per questo, nell'analisi semiotica «non si considererà nessuna informazione sull'autore, sulla sua ideologia o sulla sua competenza che non sia contenuta nel testo» ${ }^{16}$, trattato come oggetto che garantisce in sé la sua leggibilità e nel quale il significato si ottiene per mezzo di un'analisi interna al testo stesso e tramite l'osservazione dei meccanismi che producono effetti di senso che portano al 'far credere'.

Riteniamo che sia questa la posizione che meglio può aiutarci a capire e analizzare un testo come questo. L'attenzione si sposta dal 'vero' all'apparentemente tale e ci spinge ad esaminare soltanto gli effetti di senso di realtà e il modo in cui sono costruiti, invece della realtà in sé. Come abbiamo visto, gli stratagemmi linguistici e le scelte discorsive di madrelingua 'vogliono far credere' e possono riuscirci con una probabilità ancora maggiore rispetto a un testo 'tradizionale', data la rottura degli schemi e delle strutture testuali ottenuta tramite la presenza dei due narratori, cui si aggiunge la decisione di non portare a termine il romanzo lasciandolo 'a metà' e liberandosi, pertanto, della necessità di risolvere enigmi e chiarire identità.

La semiotica tratta l'autobiografia come un discorso in cui un io 'apparentemente' parla di sé, ma non si preoccupa di classificarla secondo il suo 'referente reale' contrapposto ai 'referenti immaginari' contenuti, ad esempio, in un romanzo. Ciò che importa è che, all'interno del testo,

\footnotetext{
${ }^{16}$ Jean Marie FlOCH, Alguns conceitos fundamentais em Semiótica Geral, traduzione portoghese di A. Dutra Pillar, San Paolo, Série Documentos do Centro de Pesquisas Sociossemióticas, 2001, p. 16 (la traduzione in italiano è mia).
} 
seguendo regole determinate e stabili, si costruisce il simulacro dello scrittore. Se è questo il tipo di approccio scelto, sarà, dunque, più importante esaminare le strategie utilizzate per ottenere un risultato 'verosimile' che cercare di 'verificare', confrontandolo con la realtà, ciò che si afferma.

Rientra in questo tipo di riflessione la seguente citazione di Bachtin che, nel suo famoso saggio intitolato «Le forme del tempo e del cronotopo nel romanzo», scrive:

Anche se egli ha creato un'autobiografia o una confessione assolutamente veridica, lo stesso egli, in quanto l'ha creata, resta fuori del mondo in essa raffigurato. Se io racconto (o scrivo) di un avvenimento che mi è appena successo, io in quanto lo racconto (o scrivo), mi trovo già fuori dello spazio-tempo nel quale quell'evento si è compiuto. Identificare assolutamente il mio 'io' con 1' 'io' del quale racconto è impossibile, come è impossibile sollevarsi prendendosi per i propri capelli ${ }^{17}$.

Forse è questa consapevolezza che ha spinto Julio Monteiro Martins a evitare l'autobiografia esplicita e a preferire un 'romanzo' in cui poter parlare di se stesso senza mai rivelarsi completamente e senza alcuna pretesa o impegno nei confronti della verità. Riuscire a parlare della propria vita o credere di poter creare un'immagine 'vera', definitiva ed emblematica di sé, come vorrebbe l'autobiografia, è, di fatto, un arduo (impossibile?) compito. Con la messa in scena di un secondo narratore che, da una parte, utilizza stratagemmi per convincere il lettore a credere che coincide con l'autore del romanzo e, dall'altra, si protegge allontanando da sé la necessità di assumersi un impegno, Martins crea una soluzione che si colloca in uno spazio intermedio 'al limite' tra generi e individualità e che rappresenta proprio quest'impossibilità.

Come scrive Lejeune, nei confronti di un romanzo, che si auto-dichiara finzione e nega l'identità tra autore e narratore, il lettore è più facilmente tentato a voler scoprire punte di realtà e a trovare somiglianze tra il mondo 'vero' e quello raffigurato nel testo; mentre nei confronti dell'autobiografia, che, al contrario, si dice autentica e si basa sulla coincidenza tra autore e narratore, chi legge è spinto a individuare i momenti in cui l'opera si allontana da ciò che è (stato) e a cercare le differenze, gli errori e le deviazioni. Un libro come madrelingua può permettere di fare entrambe le cose e, al tempo stesso, nessuna delle due.

\footnotetext{
${ }^{17}$ Michail BACHTIN, Estetica e romanzo, Torino, Einaudi, 1979, pp. 403-404.
} 